\title{
Stellungnahme der JHaS* zum neuen Weiter- bildungsprogramm «Allgemeine Innere Medizin»
}

\author{
Corinne Bertschi, \\ Monika Reber Feissli, \\ Miriam Schöni, \\ Sven Streit
}

\section{Zum Thema dieses}

Artikels siehe auch das Editorial von Max Giger (S. 201) sowie die Beiträge von Bruno Kissling (S. 230) und Daniel Widmer (S. 233).

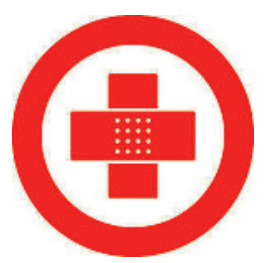

* JHaS - Die Jungen Hausärztinnen und -ärzte Schweiz bestehen seit 2006 . Der Grundgedanke ist die Vernetzung von Ärztinnen und Ärzten in der Weiterbildung zum Hausarzt. Mittlerweile sind die JHaS mit vielen Organisationen verknüpft und Mitglied von «Hausärzte Schweiz». Mehr auf www.jhas.ch

** Die im Text verwendete männliche Form schliesst Frauen mit ein.

Korrespondenz: Dr. med. Sven Streit Co-Präsident JHaS Dapplesweg 14 CH-3007 Bern sven.streit@jhas.ch

\section{Was bisher geschah}

Liebe Leserin, lieber Leser, Ihnen ergeht es wie den meisten: Sie lesen in dieser Ausgabe der SÄZ vielleicht zum ersten Mal, dass es ein neues Weiterbildungsprogramm «Allgemeine Innere Medizin» als Ersatz für den FMH Allgemeinmedizin und FMH Innere Medizin geben wird. Dieses Programm soll Mitte 2010 in Kraft treten. Wird es Sie betreffen? Ja! Denn als Hausarzt ${ }^{* *}$ haben Sie ein elementares Interesse an der Qualität und den Inhalten der Weiterbildung Ihres erhofften Praxisnachfolgers. Als Assistenzarzt werden Sie sowohl mit Berufsziel Hausarzt wie auch Spitalinternist betroffen sein, es ist schliesslich Ihre Weiterbildung. Sollten Sie Student sein und dieses Heft als ausserordentliches Mitglied der FMH erhalten, wird Sie dieses Programm noch viel mehr interessieren.

Glücklicherweise ist das vorgestellte Programm nun öffentlich [1] und Sie können lesen, was Sie erwarten soll. Versprochen wurde schliesslich viel! «Die neue Weiterbildung soll den Vorstellungen junger oder angehender Medizinerinnen und Medizinern entsprechen» [2] Wir rufen Sie also dazu auf, dieses Programm herunterzuladen und zu studieren.

Wir als Mitglieder der JHaS ${ }^{* *}$ beschäftigen uns mit der Weiterbildung seit unserer Gründung im Jahre 2006. Durch unsere aktive Teilnahme am Vasco da Gama Movement [3], der europäischen Organisation für junge und zukünftige Hausärzte, konnten wir unseren Blick über die Landesgrenze hinaus erweitern und mussten bald feststellen, dass es viele verschiedene Wege gibt, Hausarzt zu werden. Uns wurde klar, dass wir in der Schweiz kein Vorzeigemodell der Hausarztweiterbildung haben. Letztes Jahr haben wir deshalb ein Positionspapier zur Weiterbildung [4] formuliert, um unsere Erwartungen an die zukünftige Entwicklung darzulegen.

Am 4.12.2009 erhielten wir zum ersten Mal detailliert Einsicht ins vom SIWF [5] verabschiedete Programm. Beim Lesen stellten sich uns viele Fragen. Wir hörten aber auch, dass das Programm erstens nicht veröffentlicht werde bis zum Inkrafttreten am 1.7.2010 und es zweitens beschlossen und nicht mehr veränderbar sei. So verfassten wir zum Jahreswechsel eine ausführliche Stellungnahme und Auflistung unserer Fragen zuhanden des SIWF.

Die Reaktionen darauf waren gewaltig! Seitdem erreichen uns täglich ein Dutzend E-Mails mit unterstützenden - aber auch mit beschwichtigenden Worten. Dass wir nur acht Tage später zusammen mit Vertretern des SIWF an einem Tisch sitzen konnten, zeigte uns, dass wir Wichtiges hinterfragen. Unsere Kritik wurde wohlwollend entgegengenommen. Wir hörten, dass das neue Programm ein Projekt sei, das seit 1997 andaure. Viele unserer Fragen seien im Verlauf der Verhandlungen bereits geklärt worden. Allen liege viel an einer starken Hausarztmedizin und alle möchten mit dem neuen Entwurf etwas Modernes für uns, die junge Generation, entwerfen. Dabei wurden wir den Eindruck nicht los, die Hausarztmedizin werde als «Plan B» eines Arztes angeschaut. Diese Resignation entmutigt uns und ist auch im Programm spürbar. Wie wollen wir junge und zukünftige Hausärzte für einen Beruf begeistern, dessen Weiterbildung nicht attraktiv ist? Die genannte Durchlässigkeit und der modulartige Aufbau sollen denjenigen helfen, die nicht 35 Jahre lang Hausarzt bleiben wollen, um dem Anspruch der Flexibilität der jungen Generation entgegenzukommen. Grundsätzlich eine gute Idee - wenn dafür nicht die Schaffung eines klaren Profils der Hausarztmedizin geopfert würde. Schade! Hören wir doch von jungen Kolleginnen und Kollegen aus dem Ausland, wie attraktiv dort ihre Weiterbildung [6] sei. In gewissen Ländern muss man sich sogar dafür bewerben!

\section{Weniger formulieren, mehr Inhalt!}

Auf unsere Intervention hin wurden viele sprachliche und formelle, aber nur wenige inhaltliche Änderungen am Weiterbildungsentwurf vorgenommen. Zum Beispiel wurde das Wort «Praxisinternist» durch das Wort «Hausarzt» ersetzt, um nur eine Änderung zu nennen. Als inhaltliche Änderungen in eine gute Richtung möchten wir folgendes erwähnen: Praxisassistenzstellenstellen können neu bis zu einem Jahr angerechnet werden, den Weiterzubildenden wird unabhängig der Weiterbildungsstätte (Spital oder Praxis) eine minimale theoretische Weiterbildungszeit zugesichert und 6-monatige Curricula in Wahlfächern (Aufbauweiterbildung) sollen begünstigt werden. Dies alles sind Schritte in die richtige Richtung, doch nach wie vor bleiben für uns grundlegende Fragen offen.

Der Weiterbildungsentwurf hält an einer 3-jährigen Basisausbildung in Innerer Medizin (mit einem A-Jahr, maximal 6 Monate davon ambulant) fest. Diese wird gefolgt von einer 2-jährigen Aufbauweiterbildung (Wahlfächer, auch ambulant möglich), die sich nach dem angestrebten beruflichen Tätigkeitsfeld ausrichten soll. Grundsätzlich ist diese Aufbauweiterbildung sinnvoll, jedoch scheinen 2 Jahre in Anbetracht des vielfältigen Spektrums in der Hausarztpraxis dafür zu wenig. Bereits mit dem jetzigen Weiterbildungscurri- 
1 www.fmh.ch/bildung-siwf/ weiterbildung_allgemein/ weiterbildungsprogramme/ weiterbildungsprogramm allgem_doc.html

2 Bürgi U, Héritier F, Müller M. Das neue Weiterbildungsprogramm für Allgemeine Innere Medizin: modular, pragmatisch, sinnvoll. PrimaryCare. 2009;9(20):353.

3 Vasco da Gama Movement, www.vdgm.eu

4 Streit S, Ramser M. Zukünftige Hausärzte nehmen Stellung zur Ausund Weiterbildung. PrimaryCare. 2009;9(2):32-5.

5 Schweizerisches Institut für ärztliche Weiter- und Fortbildung, www.fmh.ch/ bildung-siwf.html

6 EURACT - European Academy of Teachers in General Practice «GP/FM Training Schemes in Europe» http://module.achg.be/euract

7 Workforce Studie 2006 (in Publikation). Eine repräsentative Umfrage bei 1800 Hausärzten in der Schweiz.

8 Initiative «Ja zur Hausarztmedizin» - www.jzh.ch

9 Die Europäische Definition der Hausarztmedizin (2002) beispielsweise in PrimaryCare 2003;3:261. culum zeigt sich, dass Hausärzte sich oft 8-9 Jahre lang weiterbilden [7], bevor sie in die Praxis gehen. Angesichts des Hausärztemangels und der Attraktivität der Weiterbildung erachten wir diese Entwicklung alles andere als günstig oder motivierend. Im Weiteren gilt: Grundsätzlich sind drei Jahre Praxisassistenz für den Hausarzt möglich, existieren aber im Curriculum nur fakultativ - ebenso wie eine breite Fächerung der Aufbauweiterbildung. Auch die neu geregelte theoretische Weiterbildung von 5 Stunden pro Woche (unabhängig des Weiterbildungsortes) ist bis auf eine Stunde nicht als hausärztespezifisch geregelt - auch hier bleibt für den Hausarzt Wesentliches fakultativ. Mit dem neuen Programm wird es also (immer noch) möglich sein, Hausarzt zu werden, ohne je in einer Praxis gearbeitet zu haben, mit einem Minimum an ambulanter Erfahrung (z. B. Poliklinik, Notfallstation) von 6 Monaten und ohne obligatorische hausarztspezifische, theoretische Weiterbildung. Wir empfinden dies als verpasste Chance des Weiterbildungsentwurfs, der Hausarztmedizin nicht nur auf politischer, sondern auch auf fachlicher Ebene ein klares Profil als eigenständige Disziplin zu geben. Dieser Aufbau wird viele dazu verleiten, wie in der Einführung beschrieben, vornehmlich eine internistische Weiterbildung anzustreben und, falls sich diese dann eventuell doch nicht mehr als Ziel eignet, auf Plan B, die Hausarztmedizin, umzusteigen.

Bereits vor einem Jahr haben wir in einem Positionspapier zur hausärztlichen Aus- und Weiterbildung [4] folgendes gefordert:

\section{Kernpunkte}

Das neue Weiterbildungsprogramm ist nun veröffentlicht und auch die Generation zukünftiger Spitalinternisten und Hausärzte kann konstruktiv daran mitarbeiten. Wir begrüssen die Bestrebungen der Autoren des neuen Programms, die Hausarztmedizin stärken zu wollen. Anlässlich der ausserordentlichen SIWFSitzung wurde dies von allen Teilnehmern versichert.

- Die inhaltliche Umsetzung der Weiterbildung zum Hausarzt beinhaltet jedoch noch substantielle Mängel. Bis heute fehlen klare hausarztspezifische Lernziele, welche die zukünftigen Hausärzte beherrschen müssen.

- Wir sind überzeugt, dass der Track der Hausarztmedizin über einen klaren Aufbau mit hausarztspezifischen Weiterbildungsangeboten (Praxisassistenz, spitalbegleitende Hausarztcurricula, Mentoringprogramme) - obligat und nicht nur als Plan B - verfügen muss. Ebenso müssen Karrieremöglichkeiten (z. B. Forschung, Lehre) gefördert werden. Die obligate internistische Basisweiterbildung darf nicht mehr als zwei Jahre betragen, um die breite Weiterbildung innerhalb der vorgesehenen fünf Jahre nicht zu gefährden.

- «Die Hausarztmedizin ist eine akademische und wissenschaftliche Disziplin mit eigenen Lehrinhalten, eigener Forschung, eigener Nachweisbasis und einer eigenständigen klinischen Tätigkeit. Sie ist als klinisches Spezialgebiet auf die Primärversorgung ausgerichtet» [9].

- Ausbau spitalbegleitender, fachspezifischer Weiterbildungsangebote und Praxisassistenzen

- Breite Weiterbildung

- Anerkennung der Hausarztmedizin als eigene Disziplin

Diese drei Punkte sind im aktuellen Entwurf nicht erfüllt: Praxisassistenz wird nur als wünschbare fakultative Möglichkeiten taxiert und eine hausarztspezifische, strukturierte, spitalbegleitende Weiterbildung mit garantierter Teilnahmemöglichkeit ist nicht gesichert. Damit ist die Anerkennung der Hausarztmedizin als eigene Disziplin nicht gewährleistet.

Um dies zu erreichen, müssten folgende Punkte im Weiterbildungscurriculum verankert werden:

- Mind. 1 Jahr Praxisassistenz obligatorisch für alle

- Garantierte Teilnahmemöglichkeit an strukturierten, hausarztspezifischen, spitalbegleitenden Curricula an jeder Weiterbildungsstätte

- Gewährleistung der breiten Weiterbildung durch Begünstigung von Rotationsstellen

- Reduktion der internistischen Basisweiterbildung auf maximal 2 Jahre und Verzicht auf das A-Jahr

- Gesicherte Anerkennung des neuen Titels als Hausarzt (General practitioner/Family Doctor) in Europa, ohne dass die Hausarztweiterbildung auf den Titel «praktischer Arzt» reduziert werden muss.

Auch fragen wir uns, ob eine englische MultipleChoice-Prüfung die richtige Methode darstellt, die Qualität der Hausarztmedizin zu sichern und die wichtigen Aspekte der Qualität eines Hausarztes zu erfassen.

\section{Wie geht es weiter?}

Der neu geplante Titel «Allgemeine Innere Medizin» wirft mehr Fragen auf, als dass er die Chance beinhaltet, eine qualitativ hochstehende, breite und fachspezifische Entwicklung der Hausarztweiterbildung zu fördern. Als aus Überzeugung Mittragende der Initiative «Ja zur Hausarztmedizin» [8] akzeptieren wir diesen Entwurf in der aktuellen Form nicht. Wir sind uns bewusst, dass das Plenum des SIWF den Entwurf bereits abgesegnet hat. Für die jungen Hausärzte Schweiz ist die Weiterbildung jedoch ein eminent wichtiges Thema.

Wir sehen ein, dass die Weiterbildungsprogramme immer wieder einem Wandel unterliegen und nichts für die Ewigkeit definiert wird. Trotzdem müssen wir verbindlich und kontinuierlich auf eine gute Weiterbildung zum Hausarzt hinarbeiten. Dafür kämpfen wir mit bei der Initiative «Ja zur Hausarztmedizin». Gemeinsam wollen wir die Grundversorgung stärken und ausbauen, damit wir zukünftig gute Hausärztinnen und -ärzte werden und bleiben! 